[Agr. Biol. Chem., Vol. 35, No. 1, p. 92 98, 1971]

\title{
Extracellular Accumulation of Phenazine Derivatives by Arthrobacter paraffineus Grown on $n$-Paraffin
}

\author{
By Takeo Suzuki, Kazuo Uno and Takashi DeguchI \\ Tokyo Research Laboratory, Kyowa Hakko Co. Ltd., Machida, Tokyo \\ Received June 22, 1970
}

\begin{abstract}
During the growth of Arthrobacter paraffineus KY 7134 on $n$-paraffin as the sole source of carbon, it was found that two sorts of crystalline pigments, yellow and deep-red, were accumulated in the culture medium. These were isolated and identified respectively as 1,6-dihydroxyphenazine (DHP) and 1,6-dihydroxyphenazine-5,10-di-N-oxide (DHPO).

For the production of these pigments by this microorganism, n-paraffin, particularly $n$-octadecane was the most preferable carbon source. Furthermore, controlling the medium$\mathrm{pH}$ in the neutral range and keeping the aeration of the higher level in the growing phase were essential for this fermentation.
\end{abstract}

It has been reported that several bacterial strains produced phenazine derivatives from carbohydrates. ${ }^{1 \sim 7)}$ However, little is known about the production of these compounds by hydrocarbon-grown bacteria except for that of phenazine-1-carboxylic acid. ${ }^{8}$

During the growth of Arthrobacter paraffineus KY 7134 on $n$-paraffin as the sole source of carbon, it was observed that yellow and deepred pigments were accumulated as crystalline forms in the culture medium. These two kinds of pigments were also characterized as phenazine derivatives.

1) G. R. Clemo and H. Mcllwain, J. Chem. Soc., 1938, 479.

2) Y. Sasaki, T. Yoshida and H. Sasaki, Abstracts of Papers, Annual meeting of the Agricultural Chemical Society of Japan, Tokyo, April, 1959, p. 35.

3) N. N. Gerber and M.P. Lechevalier, Biochem., 4, 176 (1965).

4) N. N. Gerber, J. Heterocyclic Chem., 6, 297 (1969).

5) N. N. Gerber, Hakko Kogaku Zasshi, 4, 176 (1964).

6) G. A. Swan and D. G. 1. Felton, Biochem., 5, 182 (1966).

7) R. Takeda and I. Nakanishi, Hakko Kogaku Zasshi, 38, 9 (1959).

8) T. Higashihara and A. Sato, Agr. Biol. Chem., 33, 1802 (1969).
The present paper is concerned with the isolation and identification of these compounds, and with the relation of the pigment production to the cell growth. In addition, the culture conditions essential for the production are described as well.

\section{EXPERIMENTAL}

Microorganism. Arthrobacter paraffineus KY 7134 was used throughout this work. This strain was isolated as one of the revertants of Arth. paraffineus KY 7104,91 an auxotrophic mutant requiring isoleucine, $\alpha$-ketobutyric or $\alpha$-aminobutyric acid.

Medium and culture condition. The medium which contains the same components as described in the previous paper10l was used in this work. The $\mathrm{pH}$ of the medium was adjusted to 7.0 before the sterilization. Usually, $20 \mathrm{ml}$ of the culture medium was poured to each $250 \mathrm{ml}$ Erlenmeyer flask. In each run, the inoculum $(1 \mathrm{ml})$ was transferred to each flask from the seed culture, which was prepared by the preincubation in the sorbitol-bouillon medium $(10 \mathrm{ml})$ for

9) K. Takayama, S. Abe and S. Kinoshita, Amino acid and Nucleic acid, 19, 121 (1969).

10) T. Suzuki, K. Tanaka, I. Matsubara and S. Kinoshita, Agr. Biol. Chem., 33, 1619 (1969). 


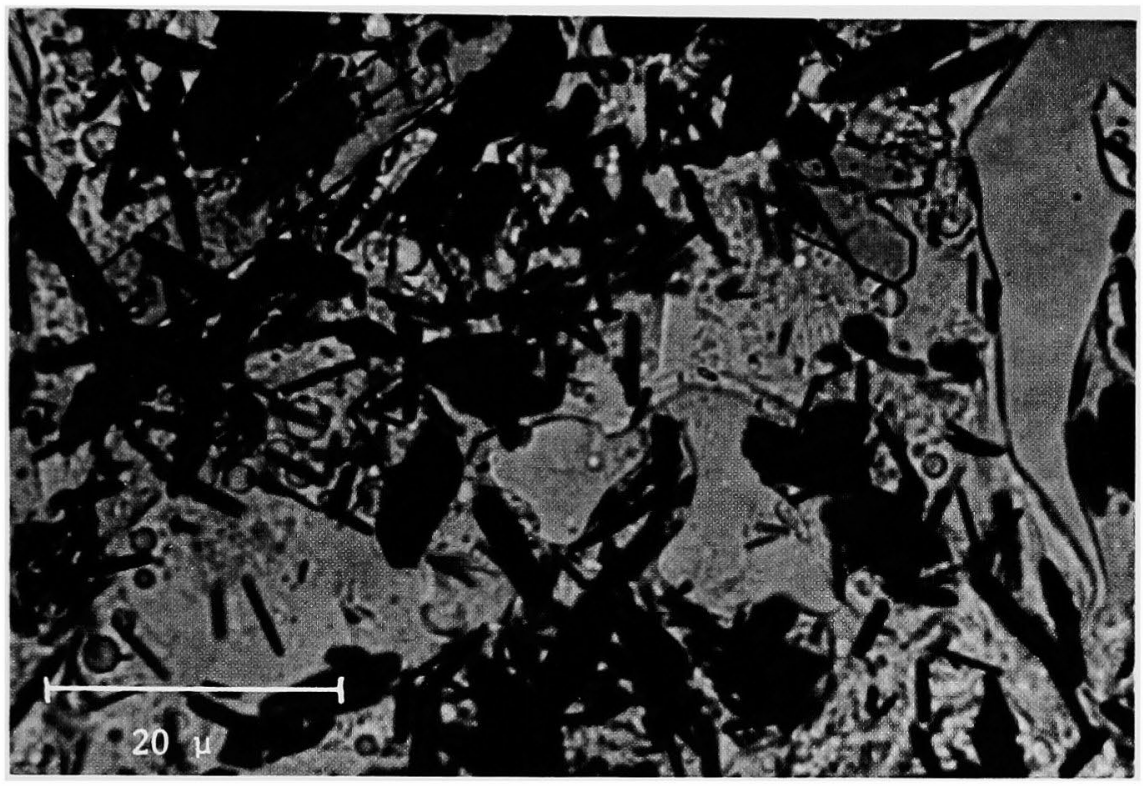

FIG. 1. Accumulation of Crystalline Pigments in the Emulsion Layer of the Culture Medium Containing $n$-Paraffin.

Cultivation for $48 \mathrm{hr}$ in 5 liter-jar fermentor.

$24 \mathrm{hr}$ at $29^{\circ} \mathrm{C}$. The fermentation was carried out at $30^{\circ} \mathrm{C}$ on the rotary shaker at $200 \mathrm{rpm}$. During the incubation, the $\mathrm{pH}$ of the medium was adjusted to $6.5 \sim 7.0$ by the occasional addition of ammonium carbonate solution. In the other experiments, 5 literjar fermentors were also employed by the same procedure as shown previously. ${ }^{10}$

Determination of cell growth. Assay was performed according to the method described in the previous paper. ${ }^{101}$

Analysis of phenazine derivatives. Two sorts of pigments produced by this microorganism were separated each other by the following procedure. Sample $(5 \mathrm{ml})$ taken out of the culture broth was extracted with chloroform $(15 \mathrm{ml})$ by vigorous shaking for $5 \mathrm{~min}$. After the centrifugation, a given volume $(30 \mu \mathrm{l})$ from the chloroform layer was subjected to paper chromatography using Toyo Roshi No. 51 A. Ascending method was employed firstly in methanol for $1 \mathrm{hr}$ and dryed paper was successively developed in chloroform for $0.5 \mathrm{hr}$. Yellow pigment (DHP) migrated with methanol and deed-red pigment (DHPO) with chloroform. Subsequently each spot thus separated on the paper was cut off and extracted with ethanol $(5 \mathrm{ml})$ in glass tubes with stopper at $50^{\circ} \mathrm{C}$ for $1 \mathrm{hr}$. The ethanol extracts were followed by the measurement of UV-absorption at $273.5 \mathrm{~m} \mu$ (for DHP) and $291 \mathrm{~m} \mu$ (for DHPO). The linear correlation was demonstrated between the quantity of phenazine derivative and the absorbancy at the respective wave length.

Other analytical procedures. Estimation of $n$-paraffin remained in the culture medium was analyzed by the previous method.101 The conventional procedures of the physicochemical analysis were applied to the identification of the pigments isolated.

\section{RESULTS AND DISCUSSION}

\section{Accumulation of crystalline pigments in culture medium}

When Arthrobacter paraffineus KY 7134 was incubated with $n$-paraffin as the sole source of carbon in 5 liter-jar fermentor, the cell 
growth occurred after the lag phase of several hours and continued for about $40 \mathrm{hr}$. In this course, it was apparently observed that the coloration of the culture broth became remarkably darker. Microscopic observation demonstrated that yellow and deep-red pigments were accumulated as crystalline forms in the emulsion layer of the culture medium as represented in Fig. 1.

Thus, the isolation and purification of these compounds were attempted as below.

\section{Isolation of crystalline pigments}

A typical isolation procedure is given below for a starting amount of $350 \mathrm{~g}$ of the emulsion layer which was obtained by the centrifuge of the whole broth (2.5 liters) after the cultivation for $48 \mathrm{hr}$. The emulsion layer holding bacterial cells, lipids and $n$-paraffin remained was extracted twice with the mixture of ethanol and ethyl ether ( $3: 1,2$ liters) at room temperature. After the filtration, the solvent vas evaporated at $40^{\circ} \mathrm{C}$ in vacuo. The viscous brown residue was washed with $n$-hexane to remove $n$-paraffin and neutral lipids, and then dissolved in diluted sodium hydroxide solution $(\mathrm{pH} 12,100 \mathrm{ml})$. Reddish purple solution thus obtained was washed with ether twice and acidified by hydrochloric acid. Subsequently yellow coloration of the acidic solution was almost completely extracted with ether. After dehydration by sodium sulfate, the extract was evaporated to dryness. The residue was boiled with benzene and filtered. Evaporation of benzene afforded yellow crystals, which were recrystallized from benzene. Golden yellow plates (pigment $\mathrm{Y}$ ) $0.62 \mathrm{~g}$ were obtained.

On the other hand, the precipitate obtained by the treatment of the emulsion layer with ethanol-ether mixture was further extracted with the chloroform-methanol $(2: 1)$ until the purple coloration was almost entirely transferred to the solvent. After the evaporation of the solvent, black gruel was obtained. This was dissolved with a small volume of $0.5 \mathrm{~N}$ sodium hydroxide and washed with ether twice. Dark blue aqueous layer was acidified by hydrochloric acid. Purple coloration of acidic solution was extracted with chloroform. After dehydration, chloroform extract was concentrated to dryness and deep-red crystals came out. These were recrystallized from chloroform. Deep-red prisms with coppery luster (pigment R) $0.64 \mathrm{~g}$ were obtained.

Both the crystalline pigments thus isolated were further purified by silicic acid column chromatography. Each pigment was dissolved in warm benzene and applied on the respective column $\left(12 \mathrm{~cm}^{2} \times 10 \mathrm{~cm}\right)$. After washing columns with small amount of benzene, methanol was firstly used as the eluant to flow out yellow pigment. Subsequently chloroform was passed through to elute purple coloration. Yellow and purple fractions were concentrated respectively in vacuo. Each concentrate was dissolved in chloroform and recrystallized; purified pigment $\mathrm{Y}(0.45 \mathrm{~g})$ and pigment $\mathrm{R}$ $(0.52 \mathrm{~g})$ were obtained.

\section{Characterization of pigments isolated}

Pigment $\mathrm{R}$ was slightly soluble in chloroform, petroleum ether, glacial acetic acid, dioxan and benzene, but not soluble in water, ethanol, nor ether. It was soluble in concentrated sulfuric acid with bluish green coloration and also in aqueous sodium hydroxide with blue coloration. Pigment $\mathrm{Y}$ was soluble in benzene, alcohol and ether. This showed green coloration with concentrated sulfuric acid and purple coloration in sodium hydroxide solution.

Both pigments represented green coloration by the reaction with ferric chloride.

Melting points and elementary analysis. Since these crystals sublimed around $200^{\circ} \mathrm{C}$ in open air, closed glass tubes were used for the measurement. Pigment $\mathrm{Y}$ decomposed at 279 to $280^{\circ} \mathrm{C}$ and pigment $\mathrm{R}$ at 233 to $234^{\circ} \mathrm{C}$. Elementary analysis demonstrated the following values, $\mathrm{C} 68.47 \%, \mathrm{H} 3.83 \%, \mathrm{~N} 13.62 \%$, 
$\mathrm{O} 14.08 \%$ for pigment $\mathrm{Y}$ and $\mathrm{C} 59.85 \%, \mathrm{H}$ $3.42 \%, \mathrm{~N} 11.20 \%$, O $26.0 \%$ for pigment $\mathrm{R}$.

$U V$-absorption spectra. Each pigment dissolved in ethanol $(0.55 \mathrm{mg} / \mathrm{ml})$ was subjected to the measurement of UV-absorption at 220 to $340 \mathrm{~m} \mu$. Pigment $\mathrm{Y}$ showed the maximum absorption at $273.5 \mathrm{~m} \mu\left(\varepsilon, 1.19 \times 10^{5}\right)$, and pigment $\mathrm{R}$ at $291 \mathrm{~m} \mu\left(\varepsilon, 2.91 \times 10^{5}\right)$. Alkaline solution of both pigments demonstrated the characteristic bathochromic shift. The $\lambda_{\max }$ of the alkaline solution of pigment $Y$ coincided with that of the neutral solution of pigment $R$. This suggests the close correlation in the chemical structure between both compounds.

IR-absorption spectra. Crystalline pigments isolated were pressed into pellets with $\mathrm{KBr}$ and analyzed in infra red spectrophotometer. Results were shown in Fig. 2.

The above findings from the solubility, the color reactions with ferric chloride, and the physicochemical analysis suggest that pigment
$\mathrm{R}$ and $\mathrm{Y}$ closely resemble crystalloiodinine $\mathrm{A}$ and $B$ which were identified respectively as 1,6-dihydroxyphenazine-di-N-oxide and 1,6-dihydroxyphenazine by Irie et al. ${ }^{11}$

Mass spectra. In order to provide a further evidence for the identification of these pigments isolated, both compounds were subjected to mass spectroscopic analysis. As shown in Fig. 3, molecular ion peaks are apparently revealed at $M / e 244$ (pigment $\mathrm{R}$ ) and at 212 (pigment $\mathrm{Y}$ ). In addition, the similar fragmentations are observed in both compounds. These results substantiate that both compounds have the same ring structure, and the peak of $M / e$ 212 could be explained by the removal of two moles of oxygen from the pigment $R$. The other fragments are also explained by proposing the structure of 1,6-dihydroxyphenazine for pigment $\mathrm{Y}$ and 1,6-dihydroxyphenazine-5,10di-N-oxides for pigment $\mathrm{R}$.

From the above data, it is concluded that two kinds crystalline pigments ( $Y$ and $R$ ) iso-

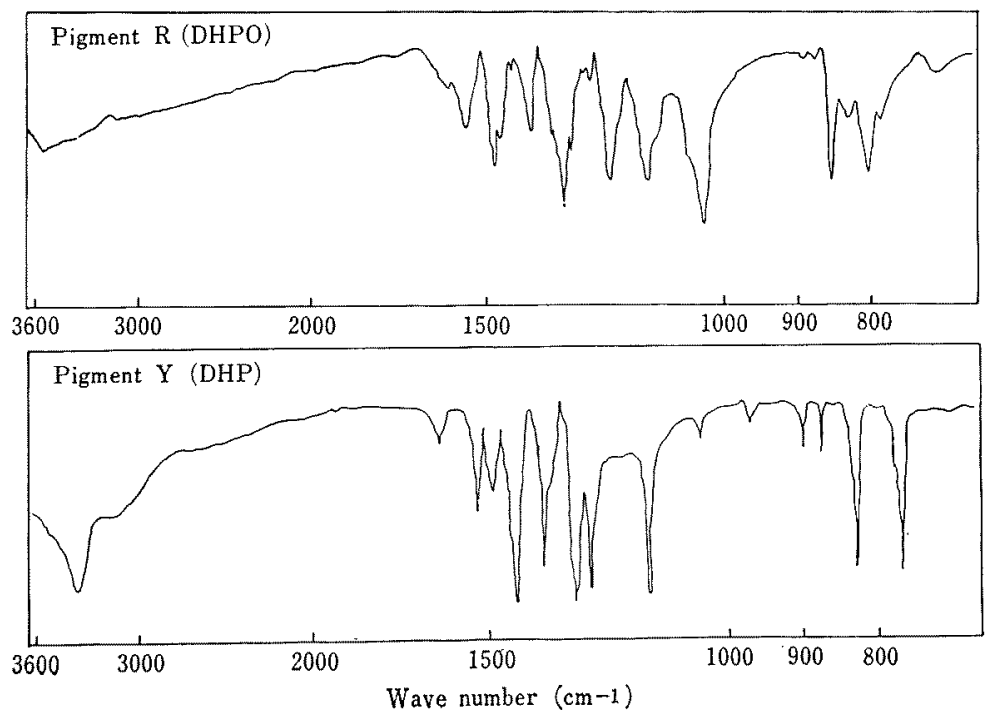

FIG. 2. IR-Spectra of Pigment $R$ and Pigment $Y$.

11) T. Irie, E. Kurosawa and I. Nagaoka, Bull. Chem. Soc. Japan, 33, 1057, (1960). 


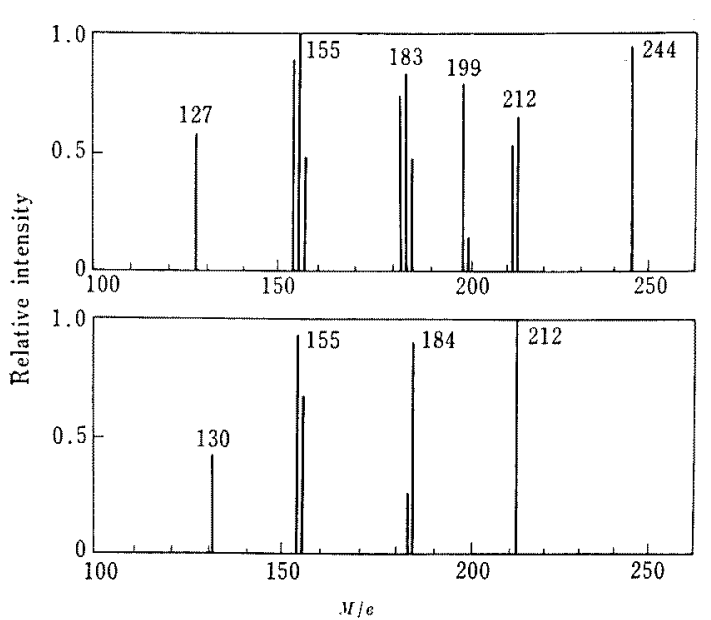

FIG. 3. Mass Spectra of Pigment R and Pigment Y. Upper, DHPO; below, DHP. Mass Spectrometer: Model CEC 110B.

lated from the culture medium of Arthrobacter paraffineus KY 7134 are identical with 1,6-dihydroxyphenazine and 1,6-dihydroxyphenazine5,10-di-N-oxide.

Culture conditions essential for pigment production

Carbon sources. According to Sasaki et al. ${ }^{21}$
Brevibacterium crystalloiodinum, when grown on carbohydrates as carbon source, produced these phenazine derivatives. In this work with Arthrobacter paraffineus, the production of these pigments was discovered firstly in the medium which $n$-paraffin mixture was used as the sole carbon source. Therefore, the other carbon sources and single fractions of $n$-paraffin were compared with it. As shown in Table I, the production of the pigments was more remarkable in the incubation with $n$-paraffin than with carbohydrate. In addition, it was apparently noted that $n$-octadecane was the most favorable carbon source for the cell growth as well as for the production of the pigments.

Nitrogen source. In the culture medium of this microorganism, ammonium phosphate has been used as inorganic nitrogen source. The other ammonium salts and some organic nitrogen sources were tested in place of ammonium phosphate. As the result, carbonate and nitrate remarkably inhibited both the bacterial growth and the production of pigments. Casamino acid, polypeptone and corn steep liquor were also suppressive to the pigment production. Although sulfate, chloride and acetate demonstrated the similar effect to phosphate, they

Table I. Effect of Various Carbon Sources on Phenazine Production

\begin{tabular}{lrrrrrr}
\multicolumn{1}{c}{ Carbon source } & Growth & DHPO & DHP & Growth & DHPO & DHP \\
Glucose & 86 & 0 & 0 & 105 & 5.0 & 27.5 \\
Sorbitol & 93 & 0 & 0 & 115 & 5.0 & 7.5 \\
$n$-Paraffin $\left(\mathrm{C}_{12} \sim \mathrm{C}_{15}\right)$ & 120 & 40.0 & 0 & 210 & 50.0 & 25.0 \\
$n$-Undecane & 50 & 10.0 & 0 & 57 & 25.0 & 25.0 \\
$n$-Dodecane & 53 & 5.0 & 0 & 210 & 35.0 & 27.5 \\
$n$-Tridecane & 58 & 12.5 & 0 & 255 & 37.5 & 20.0 \\
$n$-Tetradecane & 64 & 27.5 & 0 & 190 & 165.0 & 40.0 \\
$n$-Pentadecane & 73 & 35.0 & 0 & 195 & 120.0 & 40.0 \\
$n$-Hexadecane & 65 & 37.5 & 0 & 250 & 87.5 & 75.0 \\
$n$-Heptadecane & 115 & 60.0 & 0 & 225 & 65.0 & 40.0 \\
$n$-Octadecane & 178 & 75.0 & 0 & 250 & 250.0 & 75.0 \\
$n$-Nonadecane & 45 & 5.0 & 0 & 50 & 15.0 & -10 \\
$n$-Eicosane & 55 & 5.0 & 0 & 50 & 20.0 & 32.5 \\
\hline
\end{tabular}

Growth: O.D.260 $\times 10^{3}$, Phenazines were indicated as $\mu \mathrm{g} / \mathrm{ml}$. 
did not serve for a remarkable increase in the production yield.

Control of medium- $p H$. Since the optimal $\mathrm{pH}$ for the growth of this strain was realized in the range of 6.5 to 7.2 , it was controlled in this range by the dropwise addition of ammonium carbonate or ammonia water during the incubation. But it was not clear whether the accumulation of the pigments was owing to the occasional feeding of ammonium nitrogen to the culture medium or the control of the medium- $\mathrm{pH}$ in the neutral range. In order to elucidate this question, sodium hydroxide was used in place of ammonium salts and compared between the both cases. As shown in Fig. 4, the bacterial growth and the production of the pigments were not affected by the replacement of neutralizing agent, while both were remarkably suppressed without the control of $\mathrm{pH}$. From these results, it is apparent that the control of the medium-pH in the neutral range is essential for the production of the pigments.

Aeration. It has been known that relatively larger quantity of oxygen is required for the bacterial growth on $n$-paraffin. Therefore, the effects of the aeration on the growth and the pigment production were tested. Various volume of the culture medium $(10 \mathrm{ml}$ to $100 \mathrm{ml})$ in Erlenmeyer flasks ( $250 \mathrm{ml}$ volume) were followed by the incubation at $30^{\circ} \mathrm{C}$ for 3 days on rotary shaker $(200 \mathrm{rpm})$. The results are represented in Fig. 5. As expeted, the bacterial growth was more vigorous in the smaller

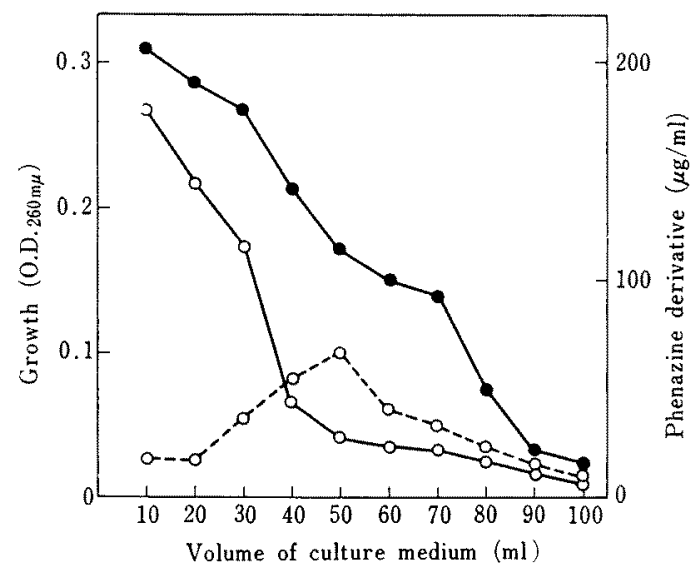

FIG. 5. Effect of Aeration on Bacterial Growth and Pigment Production.

-O, Bacterial growth; $\mathrm{O}-\mathrm{O}, \mathrm{DHPO} ; 0 \cdots \mathrm{O}$, DHP. Incubation at $30^{\circ} \mathrm{C}$ for $48 \mathrm{hr}$ on shaking.

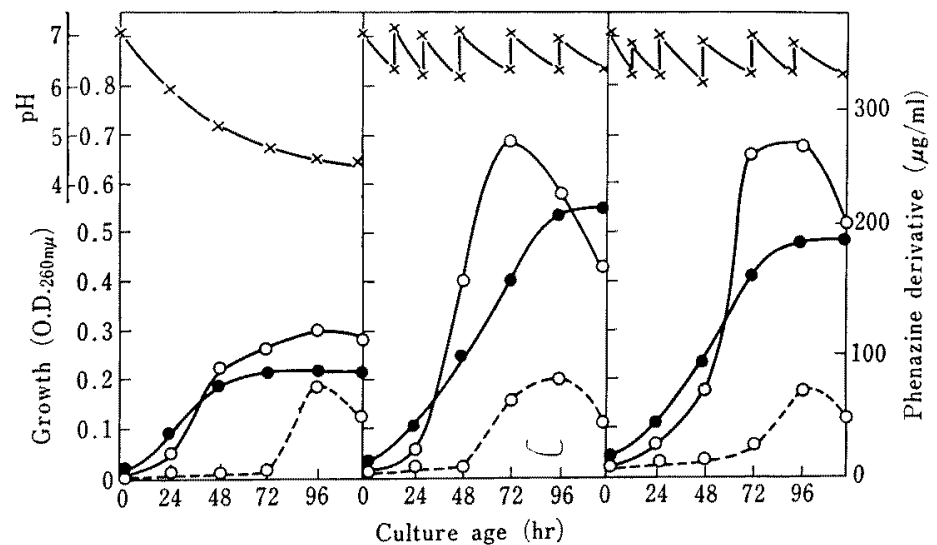

FIG. 4. Effect of pH-Control on the Production of Phenazine Derivatives in the Shaking Flasks.

-- Bacterial growth; $-0, \mathrm{DHPO}$ - $--\mathrm{O}$, DHP. Left, without $\mathrm{pH}$ control; Middle, $\mathrm{pH}$ control with $30 \%\left(\mathrm{NH}_{4}\right)_{2} \mathrm{CO}_{3}$; Right, with $4 \mathrm{~N} \mathrm{NaOH}$. 
volume of the culture media, namely in the higher aeration. The production of pigment $R$ (DHPO) was also more stimulated in the higher aeration.

\section{Time course of the pigment production}

In this experiment, 5 liters-jar fermentors were used under the same conditions as de-

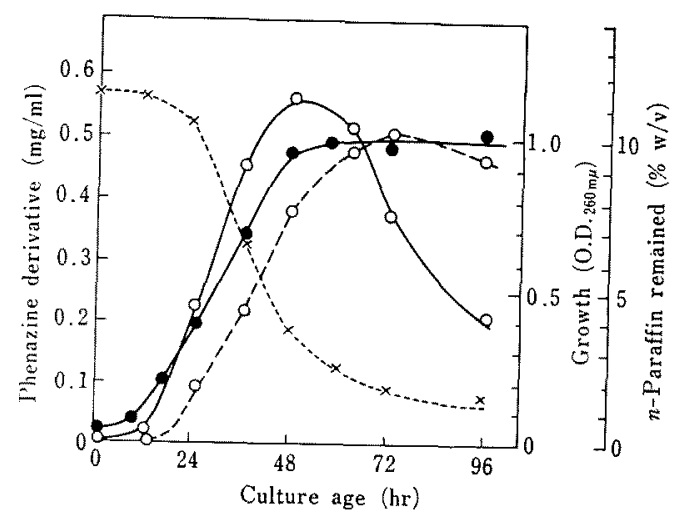

FIG. 6. Time Course of the Production of Phenazine Derivatives in 5 liter-Jar Fermentor.

-0, Bacterial growth; O-O, DHPO; O--O, DHP; $x \cdots \times, n$-paraffin remained. scribed previously. ${ }^{10)}$ A typical time course in the pigment production is represented in Fig. 6. DHPO appeared accompanying with the bacterial growth and gave the maximum at the early stationary phase, while DHP appeared later than the former and still continued to increase when DHPO began to decrease. Although the relationship of the biosynthesis between both pigments is not obvious herein, it is considered that DHPO could be synthesized via $\mathrm{DHP}^{12)}$ in this strain as well. However, the increase in the accumulation of DHP of the later phase was still remained to be explained.

In addition, it is of interest to study the possible role of these pigments in the oxidationreduction system of the $n$-paraffin-grown microorganism.

Acknowledgement. The authors thank Dr. S. Kinoshita, Director of Tokyo Research Laboratory of this company, for the encouragement throughout this work, and Dr. K. Tanaka and Dr. I. Matsubara for their valuable advices and discussions. Mr. H. Honda and H. Baba are also acknowledged for their assistance.

12) M. Podojil and N. N. Gerber, Biochem., 6, 2701 (1967). 